\title{
Intradiscal Injection of Autologous Platelet-Rich Plasma Releasate to Treat Discogenic Low Back Pain: A Preliminary Clinical Trial
}

\author{
Koji Akeda ${ }^{1}$, Kohshi Ohishi ${ }^{2}$, Koichi Masuda ${ }^{3}$, Won C. Bae ${ }^{4}$, Norihiko Takegami ${ }^{1}$, \\ Junichi Yamada ${ }^{1}$, Tomoki Nakamura ${ }^{1}$, Toshihiko Sakakibara ${ }^{5}$, Yuichi Kasai ${ }^{5}$, Akihiro Sudo ${ }^{1}$ \\ ${ }^{1}$ Department of Orthopaedic Surgery, Mie University Graduate School of Medicine, Tsu, Japan \\ ${ }^{2}$ Blood Transfusion Service, Mie University Hospital, Tsu, Japan \\ ${ }^{3}$ Department of Orthopaedic Surgery, University of California, San Diego, CA, USA \\ ${ }^{4}$ Department of Radiology, University of California, San Diego, CA, USA \\ ${ }^{5}$ Department of Spinal Surgery and Medical Engineering, Mie University Graduate School of Medicine, Tsu, Japan
}

\section{Study Design: Preliminary clinical trial.}

Purpose: To determine the safety and initial efficacy of intradiscal injection of autologous platelet-rich plasma (PRP) releasate in patients with discogenic low back pain.

Overview of Literature: PRP, which is comprised of autologous growth factors and cytokines, has been widely used in the clinical setting for tissue regeneration and repair. PRP has been shown in vitro and in vivo to potentially stimulate intervertebral disc matrix metabolism.

Methods: Inclusion criteria for this study included chronic low back pain without leg pain for more than 3 months; one or more lumbar discs (L3/L4 to L5/S1) with evidence of degeneration, as indicated via magnetic resonance imaging (MRI); and at least one symptomatic disc, confirmed using standardized provocative discography. PRP releasate, isolated from clotted PRP, was injected into the center of the nucleus pulposus. Outcome measures included the use of a visual analog scale (VAS) and the Roland-Morris Disability Questionnaire (RDQ), as well as X-ray and MRI (T2-quantification).

Results: Data were analyzed from 14 patients (8 men and 6 women; mean age, 33.8 years). The average follow-up period was 10 months. Following treatment, no patient experienced adverse events or significant narrowing of disc height. The mean pain scores before treatment (VAS, 7.5 $\pm 1.3 ; \mathrm{RDQ}, 12.6 \pm 4.1$ ) were significantly decreased at one month, and this was generally sustained throughout the observation period (6 months after treatment: VAS, 3.2 $\pm 2.4, \mathrm{RDQ} ; 3.6 \pm 4.5$ and 12 months: VAS, 2.9 $\pm 2.8 ; \mathrm{RDQ}, 2.8 \pm 3.9 ; p<0.01$, respectively). The mean T2 values did not significantly change after treatment.

Conclusions: We demonstrated that intradiscal injection of autologous PRP releasate in patients with low back pain was safe, with no adverse events observed during follow-up. Future randomized controlled clinical studies should be performed to systematically evaluate the effects of this therapy.

Keywords: Intervertebral disc degeneration; Platelet-rich plasma; Low back pain; Clinical trial

Received Sep 23, 2016; Revised Nov 6, 2016; Accepted Nov 29, 2016

Corresponding author: Koji Akeda

Department of Orthopaedic Surgery, Mie University Graduate School of Medicine,

2-174 Edobashi, Tsu City, Mie 514-8507, Japan

Tel: +81-59-231-5022, Fax: +81-59-231-5211, E-mail: k_akeda@clin.medic.mie-u.ac.jp 


\section{Introduction}

Recent large, epidemiological studies have indicated that low back pain is significantly associated with lumbar disc degeneration [1]. Intervertebral disc degeneration is clinically characterized by decreased signal intensity on T2weighted magnetic resonance imaging (MRI). Biochemical characteristics of degenerated intervertebral discs (IVDs) include degradation of the extracellular matrix, with loss of proteoglycan and water content in the nucleus pulposus (NP) and collagen degeneration in the annulus fibrosus (AF). These degenerative changes may lead to internal disc disruptions, including radial and circumferential tears, cracking, and fissuring, all of which are associated with pain generation [2]. Importantly, a degenerated IVD has limited potential for self-repair, which is different from the wound-healing process of other blood flow-rich tissues such as skin [3]. Abnormal tissue remodeling processes identified with disc disruptions, including the ingrowth of vascularized granulation tissue and nociceptive nerve fibers, are implicated in causing pain [4]. The pathomechanism of discogenic low back pain is generally attributed to IVD degeneration, which involves the enhanced expression of proinflammatory cytokines, neurotrophins, and the formation of internal disc disruptions [5].

In response to cellular activation, platelets can release the contents of intracellular granules, which include growth factors, coagulation proteins, adhesion molecules, cytokines, and inflammatory molecules, which are known to promote wound healing [6]. Platelet-rich plasma (PRP), which contains autologous growth factors and cytokines, has been widely used clinically for tissue regeneration and repair [6-8]. PRP has also recently been experimentally applied to degenerated IVDs to enhance self-repair [9]. However, intradiscal injection of PRP and its effect on disc regeneration has been met with controversy [10-12] because of the possibility that the injected PRP might release growth factors and/or cytokines in an unstable manner and at different rates [9].

We previously demonstrated in vitro that the soluble releasate isolated from PRP (PRP releasate) stimulated matrix metabolism of porcine IVD cells [13]. Furthermore, intradiscal injection of autologous PRP releasate has been shown to induce restoration of structural changes in vivo using the rabbit annular puncture model [14]. Therefore, we hypothesized that intradiscal injection of bioactive soluble factors (releasate) isolated from activated PRP exerts a direct effect on stimulating the repair of degenerated discs of patients with discogenic low back pain. The aim of our study was to evaluate the safety and initial efficacy of intradiscal injection of autologous PRP releasate to treat discogenic low back pain. Herein, we discuss the results of our prospective, preliminary clinical study in 14 patients with low back pain associated with lumbar disc degeneration (Pfirrmann disc degeneration grades [15]: III-IV) at L4/L5 to L5/S1.

\section{Materials and Methods}

\section{Study design}

This study was a prospective clinical feasibility study, primarily a safety assessment, conducted between April 2009 and March 2012. This study was approved by the Ethics Committee of Mie University Hospital. All participants provided informed consent.

\section{Patients}

Patients who were receiving diagnostic discography for suspected discogenic low back pain from April 2009 to March 2012 were recruited. Among 27 patients who received diagnostic discography, 14 were included in this study. Inclusion criteria for this study were being older than 18 years and having (1) chronic low back pain without leg pain for more than 3 months, (2) one or more lumbar discs (L3/L4 to L5/S1) with evidence of degenerative changes as per MRI (disc degeneration was defined as more than grade III via the Pfirrmann disc degeneration grade/classification system [15]), (3) maintenance of 50\% or more of normal disc height, and (4) at least one symptomatic disc confirmed using standardized provocative discography and/or disc block. Exclusion criteria included abnormal neurological symptoms (e.g., radiculopathy) with lumbar spinal stenosis or spondylolisthesis and inflammatory arthritis (e.g., discitis).

\section{Diagnosis of discogenic low back pain}

Provocative discography and subsequent disc block were performed to diagnose discogenic low back pain as previously reported [16], with some modifications. Spinal needles (22 gauge, 150 mm; Hakko, Chikuma, Japan) were inserted into the center of the NP under fluoroscopy. 
Radiocontrast agent (Ominipaque 240, Daiichi-Sankyo, Tokyo, Japan) was injected into the disc until pain was provoked (maximum $2.0 \mathrm{~mL}$ ). After concordant pain was evaluated, $1.0 \mathrm{~mL}$ of $2 \%$ lidocaine (AstraZeneca, Osaka, Japan) was injected. Two and 24 hours later, the extent of pain reduction was assessed. The patients with concordant pain at discography and/or improved back pain after the disc block were diagnosed with discogenic low back pain.

\section{4. $P R P$ releasate preparation}

PRP isolation was performed using a sterile blood collection bag system (KARMI Blood Bag, Kawasumi Laboratories, Inc., Tokyo, Japan) at the Blood Transfusion Service of Mie University Hospital. Whole blood (200 mL) with an anti-coagulant was first centrifuged at 3,000 $\times \mathrm{g}$ for 15 minutes at room temperature to form a buffy coat (BC) layer containing platelets and leukocytes. The BC layer was resuspended in $20 \mathrm{~mL}$ of plasma and then centrifuged at $180 \times \mathrm{g}$ for 15 minutes to separate the platelets from leukocytes and residual red blood cells. The resulting supernatant (PRP) was transferred to a storage bag (Fig. 1A). Autologous serum was prepared from $10 \mathrm{~mL}$ of coagulated whole blood by centrifuging for 10 minutes at $3,000 \times$ g. A mixture of autologous serum and $2 \% \mathrm{CaCl}_{2}$ (Otsuka, Tokyo, Japan) was added to the PRP for clot (gel) formation. After incubation for 60 minutes at room temperature, the supernatant (PRP releasate) was isolated from the PRP gel by centrifugation $(3,000 \times \mathrm{g}$ for $5 \mathrm{~min}$ utes) (Fig. 1B). The samples were kept at $-20^{\circ} \mathrm{C}$ until used.
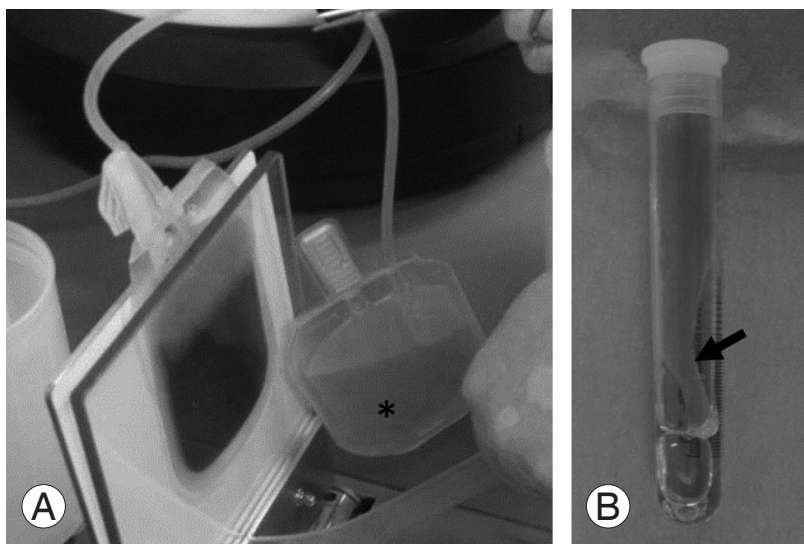

Fig. 1. Preparation of platelet-rich plasma (PRP) releasate. (A) Following the two-stage centrifugation of whole blood, PRP (asterisk) was transferred to a storage bag using a sterile blood collection bag system. (B) A mixture of autologous serum and $2 \% \mathrm{CaCl}_{2}$ was added to PRP for clot (gel) formation (arrow).
The number of platelets and white blood cells (WBCs) of each whole blood and PRP fraction was counted using a hemocytometer. The levels of platelet-derived growth factor $\mathrm{BB}$ (PDGF-BB) in the PRP releasate and autologous serum were assessed by enzyme-linked immunosorbent assay kits (R\&D systems, Minneapolis, MN, USA).

\section{Procedure for injection of PRP releasate}

Intravenous antibiotics were administrated within 60 minutes before the injection procedure. The injection site was treated with local anesthetic ( $0.5 \%$ lidocaine). Under fluoroscopy, a 22 gauge, 150-mm spinal needle was inserted into the center of the targeted disc (Fig. 2). PRP releasate, $2 \mathrm{~mL}$, was injected through a syringe filer (Millex GV Filter unit, Cat. \#SLGV M33 RS, Millipore, Billerica, MA, USA).

After injecting PRP releasate, patients were hospitalized for 12 hours and were discharged a day later. Returning to normal, daily living activities was permitted three days after discharge. Physical activity, such as sports, was permitted four weeks after treatment according to the extent of pain reduction. To more accurately evaluate the preliminary efficacy of this treatment on pain, only the temporary use of nonsteroidal anti-inflammatory drugs (NSAIDs) was permitted for unbearable pain.

\section{Efficacy assessment}

The efficacy of this treatment was assessed by a visual
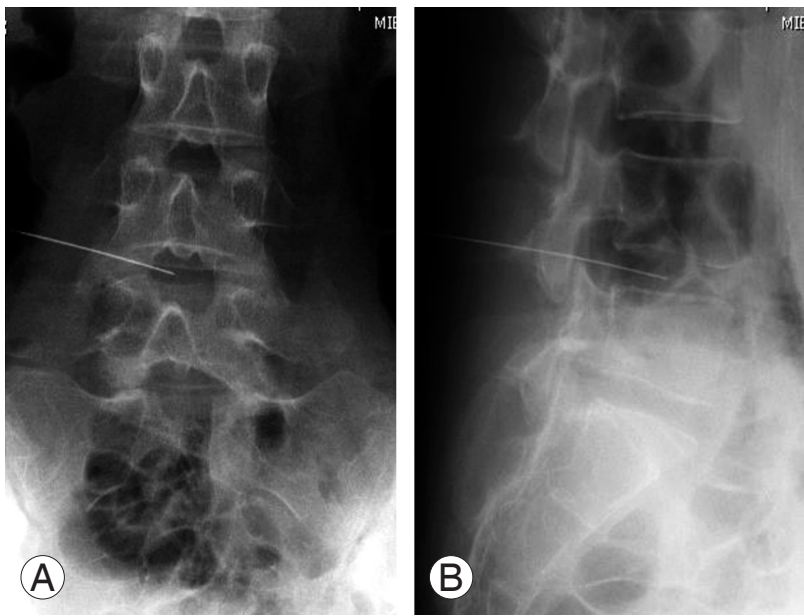

Fig. 2. Lumbar radiograph (A, anteroposterior view; $\mathbf{B}$, lateral view) at the injection site of platelet-rich plasma releasate. Under fluoroscopy, a spinal needle was inserted into the center of the targeted disc (L4/L5). 
analog scale (VAS) [17] for back pain and the RolandMorris Disability Questionnaire (RDQ) [18] for back pain-related disability at baseline and at $4,8,16,24,32$, 40 , and 48 weeks after the treatment. A neurological assessment, including motor strength, sensory function, and reflexes, was also performed at the same time points.

\section{Radiographic evaluation of the lumbar spine}

Lateral lumbar spine radiographs of each patient were taken centered on the L3 vertebrae in the standing position before and after treatment as well as every second month until the end of the study. The anterior and posterior heights and the depths of the intervertebral discs were measured, and disc height index (DHI) was calculated as previously reported [19]. The \% DHI was calculated as the rate of change in DHI compared to the baseline [(DHI at follow-up-DHI at baseline)/DHI at baseline $\times 100 \%$. The lumbar lordosis angle (the angle between the planes of the superior end plate of the first lumbar vertebra and the superior line of the sacrum) was also determined.

\section{MRI analysis}

MRI analysis was performed before treatment and at three to four months (early stage) and 12 months (late stage) after treatment. MRI was performed using a 3.0-Tesla scanner (Achieva 3.0T, Philips, Amsterdam, Netherlands) with a SENSE-Spine coil (Philips). Sagittal T2-weighted (time to repeat, or TR, of 2,500 milliseconds; time to echo, or $\mathrm{TE}$, of 90 milliseconds) fast spin-echo images were used to classify lumbar discs into five grades of degeneration using the Pfirrmann disc degeneration grading scheme [15].

Quantitative T2 mapping was performed using a multiecho spin-echo sequence in the sagittal plane. Scanning parameters were $\mathrm{TR}=2,500$ milliseconds; $\mathrm{TE}=15$ to 300 milliseconds ( $15 \mathrm{TEs}$ ); field of view $=280 \mathrm{~mm}$; slice thickness $=4 \mathrm{~mm}$; image matrix $=512 \times 512$; number of excitations $=1$; and total scanning time $=7$ minutes and $20 \mathrm{sec}$ onds. To automatically segment and analyze regions of the NP usually unapparent in degenerate discs, a templatebased method [20] was used. Briefly, MRI data from cadaveric human spines containing grade 1 discs $(n=11)$ with visibly distinct NP regions were analyzed using Otsu's method [21] to threshold and segment NPs. Individual masks of the NP and whole disc were registered (rigid body with scaling) and averaged to create a single template. The template was applied to all discs by affine registration of the template to match the mask of the target discs. The registered template was applied to T2 maps to determine average $\mathrm{T} 2$ values of the $\mathrm{NP}$ and $\mathrm{AF}$ from each disc. To reduce variability between patients, all T2 values were normalized to the values at $\mathrm{L} 3 / 4$, which were usually much less degenerate compared to the injected levels. Among the 14 patients, quantitative T2-mapping analysis was performed in 11 patients who provided consent (early stage, $n=4$; late stage, $n=1$; and both early; and late stages, $\mathrm{n}=6$ ).

\section{Safety assessments}

The safety of this treatment was evaluated in terms of neurological changes; radiological examination, including changes in disc height; the lumbar lordosis angle; and the MRI T2 value. The presence or absence of adverse events associated with this treatment was also evaluated throughout the follow-up period.

\section{Statistical analysis}

The optimal sample size of this study was estimated to provide at least $80 \%$ power to detect the differences of VAS and RDQ between pre- and post-treatment at a $5 \%$ level of significance (with 0.8 effect size) using the $G^{*}$ Power 3 program [22]. Differences in VAS and RDQ scores, MRI T2-values, and radiographic L1-S angles were assessed for statistical significance using the paired $t$ test. The significance of differences among mean radiograph measurements was analyzed by a 2-way repeated measures analysis of variance (ANOVA). The data are expressed as the mean \pm standard deviation of the mean (SD). Statistical analysis was performed using the StatView program (Abacus Concepts, Berkeley, CA, USA) with a significance level of $p<0.05$.

\section{Results}

\section{Patient population}

Data were analyzed from 14 patients ( 8 men and 6 women; mean age, 33.8 years; age range, 25-46 years). Fourteen discs were studied in this cohort; patient characteristics are summarized in Table 1. The average follow-up period was 10 months. All patients (100\%) met the follow- 
Table 1. Patient characteristics

\begin{tabular}{|c|c|c|c|c|c|c|}
\hline Patient ID & Age (yr) & Sex & Occupation & VAS (baseline) & RDQ (baseline) & MRI (Phirmman grade) \\
\hline \#01 & 44 & Male & Office worker & 7 & 11 & III \\
\hline \#02 & 27 & Female & Nurse & 8 & 12 & III \\
\hline \#03 & 26 & Male & Nurse & 7 & 12 & III \\
\hline \#04 & 45 & Male & Police officer & 8 & 8 & III \\
\hline \#05 & 25 & Female & Physical therapist & 8 & 17 & IV \\
\hline \#06 & 39 & Female & Secretary & 5 & 8 & III \\
\hline \#07 & 25 & Male & Worker & 9 & 15 & III \\
\hline \#08 & 40 & Male & Designer & 7 & 13 & III \\
\hline \#09 & 27 & Female & Gardener & 9 & 16 & IV \\
\hline$\# 10$ & 31 & Male & Cook & 7 & 10 & III \\
\hline$\# 11$ & 46 & Male & Teacher & 8 & 15 & III \\
\hline$\# 12$ & 44 & Male & Worker & 9 & 22 & III \\
\hline$\# 13$ & 27 & Female & Worker & 8 & 10 & III \\
\hline$\# 14$ & 27 & Female & Waitress & 5 & 6 & III \\
\hline
\end{tabular}

VAS, visual analog scale; RDQ, Roland-Morris Disability Questionnaire; MRI, magnetic resonance imaging.

up examination from baseline to 6 months. Ten patients (71.4\%) were available for the follow-up examination at 8 and 10 months, and 9 patients (64.3\%) were available for the final examination at 12 months. Between 6 and 8 months, four patients were lost to follow-up. Between 10 and 12 months, one patient was lost to follow-up due to relocation. The targeted discs were L4/L5 (in 11 cases) and L5/S1 (in 3 cases). The Pfirrmann disc degeneration grade was 3 in 12 patients and grade 4 in two patients. Thirteen patients received a single intradiscal injection of PRP releasate; however, one patient (\#09) received a second injection 12 weeks after the first injection because of persistent low back pain. During the follow-up period, seven patients temporarily used NSAIDs (50\%).

\section{Quality assessment of PRP and PRP releasate}

The mean platelet count of PRP was approximately 3.7 times greater than that of whole blood (whole blood, $[242.7 \pm 45.3] \times 10^{3}$ platelets $/ \mu \mathrm{L}$; PRP, $[907.1 \pm 1,039.3] \times 10^{3}$ platelets $/ \mu \mathrm{L}$ ). The mean WBC count of PRP was about $1 / 230$ of whole blood (whole blood, $[6.27 \pm 1.16] \times 10^{3}$ cells/ $\mu \mathrm{L}$; PRP, $[0.20 \pm 0.31] \times 10^{3}$ cells $\left./ \mu \mathrm{L}\right)$. The average level of PDGF-BB in the PRP releasate was approximately 2.1 times higher than that in autologous serum (PDGF-BB $[\mathrm{ng} / \mu \mathrm{L}]$, autologous serum, $3.39 \pm 1.67$; PRP, 7.12 \pm 3.11 ; $p<0.01)$.

\section{Measures of efficacy (VAS and RDQ scores)}

More than $50 \%$ reduction of LBP, as evaluated by VAS scores, was observed in $71 \%(10 / 14)$ of patients within four weeks after PRP-releasate injection; this was generally maintained throughout the observation period. However, LBP returned in two patients (\#02 and \#07) (Fig. 3A). The mean pain score (VAS) significantly decreased after injection of PRP releasate (baseline, $7.5 \pm 1.3$ [n=14]; 4 weeks, $3.1 \pm 2.5$ [ $\mathrm{n}=14]$; 8 weeks, $3.2 \pm 2.0$ [ $\mathrm{n}=14$ ]; 16 weeks, $3.4 \pm 1.9$ [ $\mathrm{n}=14] ; 24$ weeks, $3.2 \pm 2.4[\mathrm{n}=14]$; 32 weeks, $3.0 \pm 1.9$ [n=10]; 40 weeks, $2.8 \pm 2.6$ [n=10]; 48 weeks, $2.9 \pm 2.8$ [n=9]; all $p<0.01$ vs. baseline) (Fig. 3B).

Improvement in physical disability scores (RDQ scores) was relatively better than that in VAS scores. Particularly, $79 \%$ of patients (11/14) showed a significant reduction (more than 50\%) in RDQ scores four weeks after PRPreleasate injection. This was maintained for 48 weeks after treatment. However, RDQ scores returned to higher levels in the same two patients whose VAS scores rebounded (patients \#02 and \#07) (Fig. 4A). The mean RDQ score significantly decreased after injection (baseline, 12.6 \pm 4.1 [ $\mathrm{n}=14]$; 4 weeks, $5.1 \pm 5.2$ [ $\mathrm{n}=14]$ ] 8 weeks, $5.2 \pm 5.5[\mathrm{n}=14] ; 16$ weeks, $4.7 \pm 4.5[\mathrm{n}=14] ; 24$ weeks, $3.6 \pm 4.5[\mathrm{n}=14]$; 32 weeks, $4.0 \pm 3.0[\mathrm{n}=10]$; 40 weeks, $3.8 \pm 5.3[\mathrm{n}=10]$; 48 weeks, $2.8 \pm 3.9$ [n=9]; all $p<0.01$ vs. baseline) (Fig. 4B). 


\section{Radiographic assessment}

For all patients, their lumbar lateral radiographs showed no significant progression of disc height narrowing and ossification following the injection of PRP releasate into the targeted disc (Fig. 5). The \% DHI of PRP releasate-

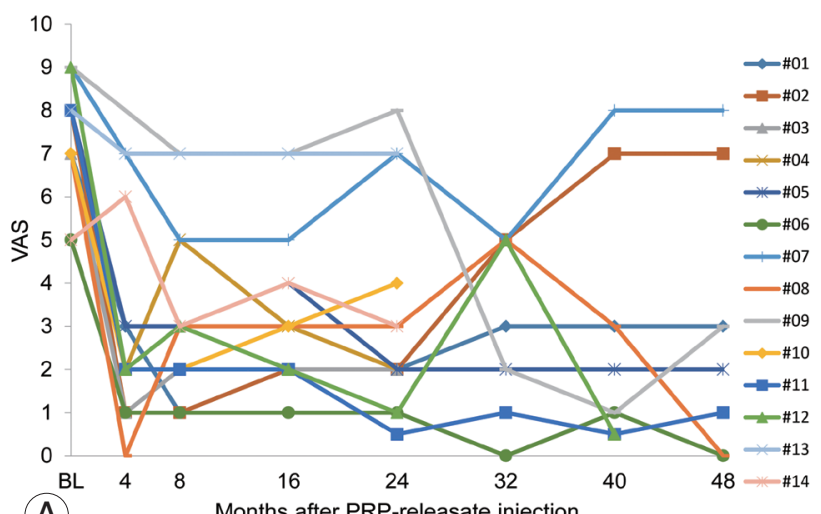

A Months after PRP-releasate injection

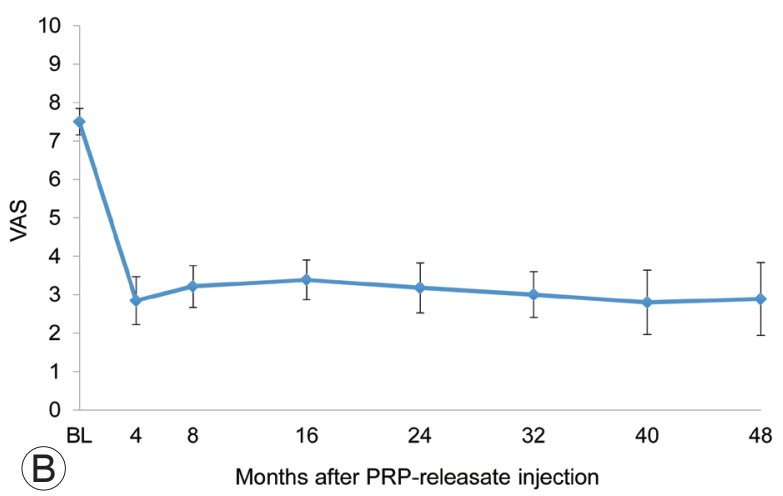

Fig. 3. Visual analog scale (VAS) scores of individual patients (A) and mean VAS scores (B) before and after intradiscal injection of plateletrich plasma (PRP) releasate. injected discs and the control (L3/L4) discs had similar changes during the follow-up period ( $p=0.9$ ) (Fig. 6A). Analysis of variance revealed no significant interaction between disc treatment and time point $(p=0.9)$. In addition, the angle of lumbar lordosis (L1-S angle) did not change significantly during the evaluation period (Fig. 6B).

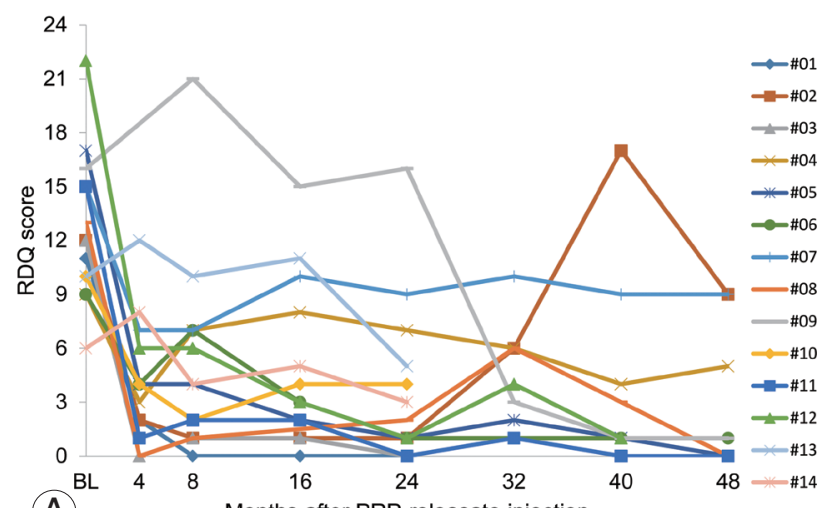

(A)

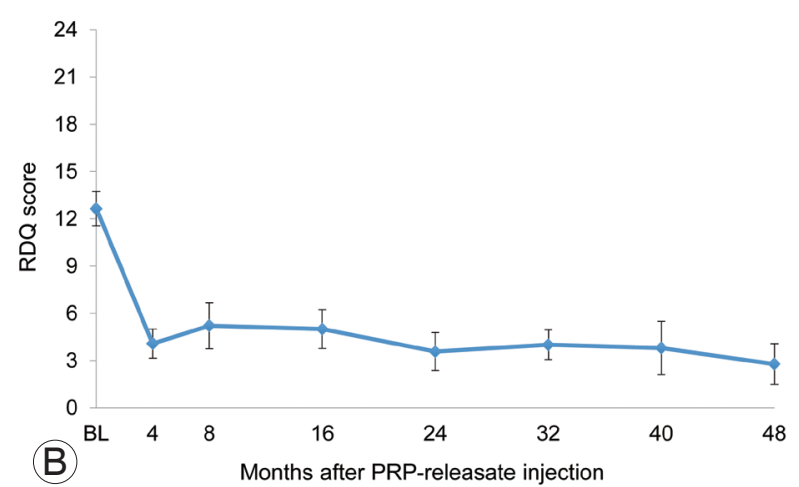

Fig. 4. Roland-Morris disability questionnaire (RDQ) scores of individual patients ( $\mathbf{A})$ and mean RDO scores $(\mathbf{B})$ before and after intradiscal injection of platelet-rich plasma (PRP) releasate.
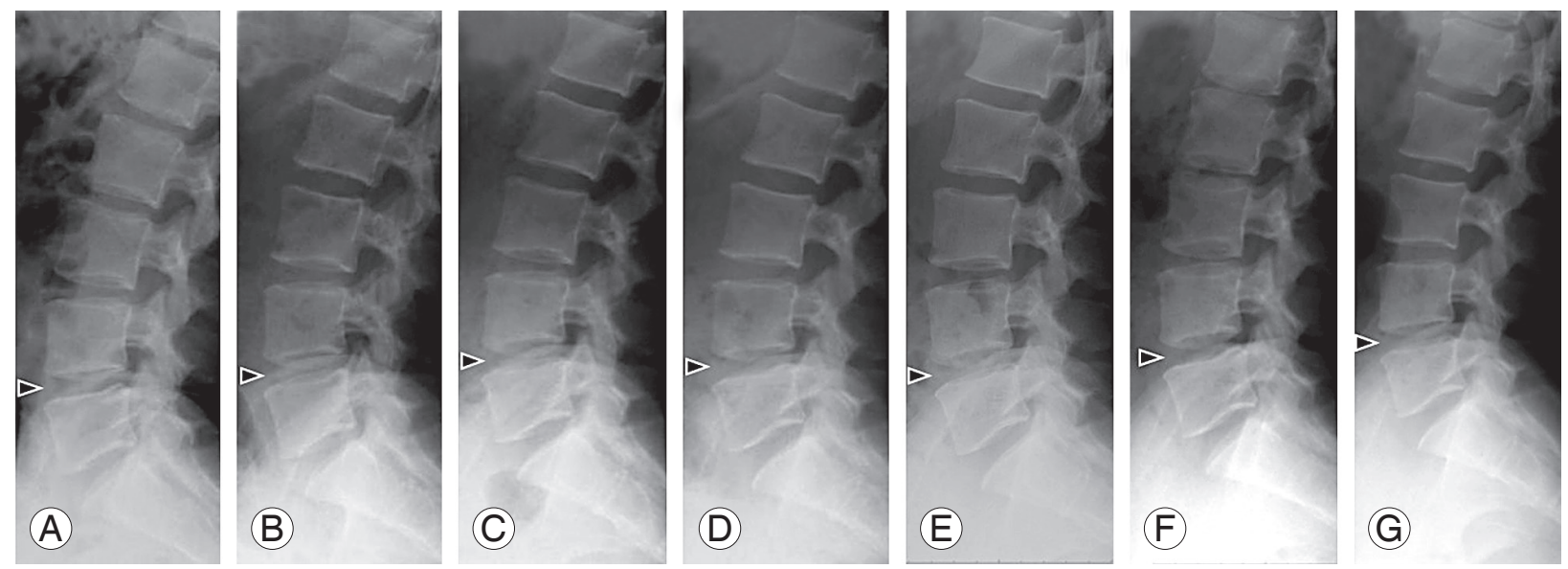

Fig. 5. Representative lumbar radiographs before and after the intradiscal injection of platelet-rich plasma (PRP) releasate. Lumbar lateral radiographs of patient \#06. Pre-treatment (A), two (B), four (C), six (D), eight (E), $10(F)$, and 12 (G) months after injection of PRP releasate. 


\section{Quantitative MRI assessment}

Sagittal T2 maps suggested no significant changes in the normalized T2 values of the NPs between baseline (Fig. 7A) and the follow-ups (early stage shown) (Fig. 7B). At baseline, the mean normalized T2 value of the NP at the treated level was $0.56 \pm 0.1$. Little change was seen at the early stage $(0.53 \pm 0.06)$, or the late stage $(0.56 \pm 0.10)$, using repeated measurement ANOVA ( $p=0.68)$ (Fig. 7C). The mean normalized T2 value of the AF showed a similar trend with no significant difference before and after treatment (baseline, 1.01 \pm 0.06 ; early stage, $1.01 \pm 0.15$; late stage, $0.94 \pm 0.09 ; p=0.35$, repeated measurement ANOVA) (Fig. 7D).

\section{Adverse events}

Two patients exhibited leg numbness at 1 month (\#04) and 6 months (\#03) post-treatment; however, this symptom disappeared within 7 days. None of the patients exhibited neurological deterioration or symptoms of discitis.
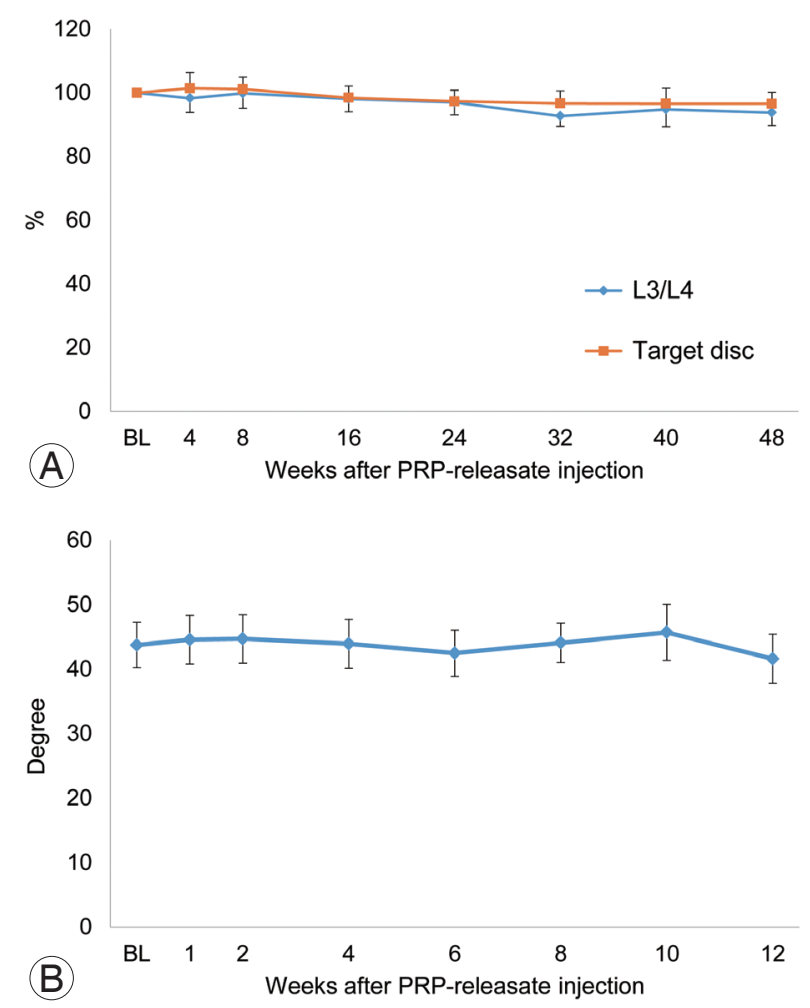

Fig. 6. Radiographic assessments. (A) Change in disc height. The \% $\mathrm{DH}$ of PRP releasate-injected discs and the control (L3/L4) discs had similar changes during the follow-up period. (B) Change in the angle of lumbar lordosis (L1-S angle). BL, baseline; DHI, disc height index; PRP, platelet-rich plasma.
No other adverse events associated with treatment were observed. Two patients (\#02 and \#07) experienced the return of low back pain, albeit not worse than that at baseline.

\section{Discussion}

In this study, we evaluated the safety and initial efficacy of intradiscal injection of autologous PRP releasate for patients with discogenic low back pain. No apparent adverse effects were identified during the follow-up period. Treatment with PRP releasate significantly improved low back pain as evaluated via VAS and RDQ scores. Radiographic analyses, including lumbar radiography and/or MRI, showed no significant changes after PRP-releasate injection compared to baseline.

PRP has been used to treat musculoskeletal pathologies [6], and particularly, there have been recent applications in orthopedic surgery, especially regarding sports-related injuries $[7,8]$. Although a number of basic research studies have shown the stimulatory effect of PRP on tissue
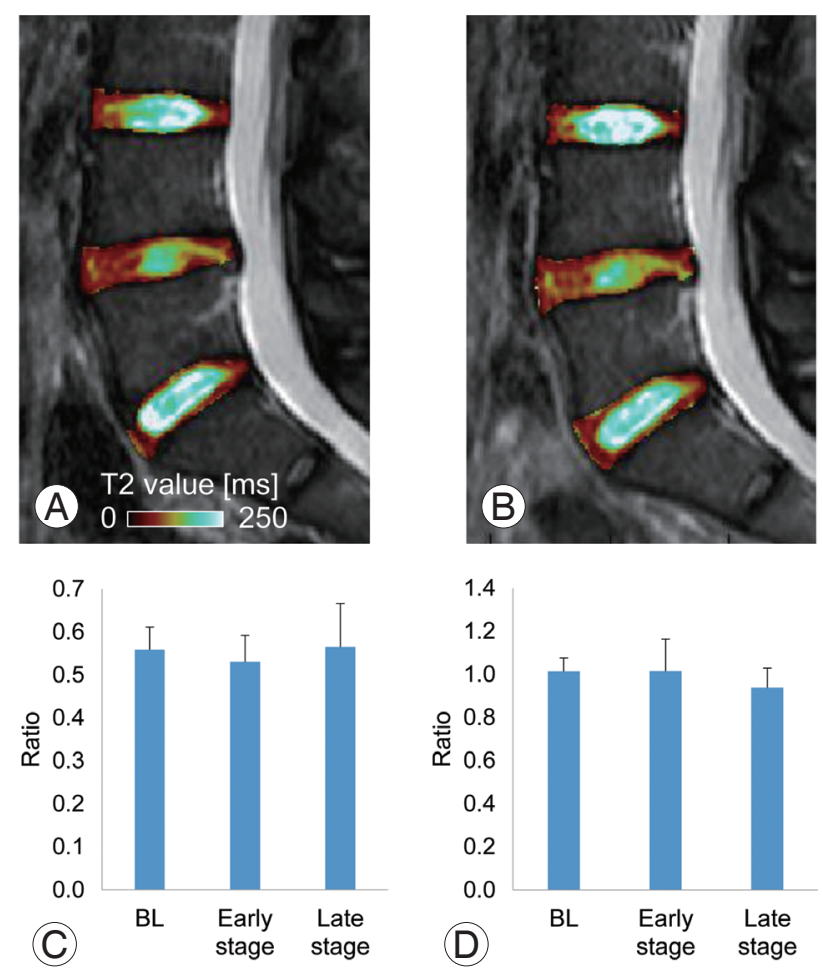

Fig. 7. Quantitative magnetic resonance imaging $T 2$ values. Representative sagittal T2 maps at baseline (A) and the early stage (B). There were no significant changes in normalized $\mathrm{T} 2$ values between those at baseline (BL) and follow-ups of both nucleus pulposus (C) and anulus fibrosus (D) tissues. 
repair, there has been a wide range of outcomes among clinical studies. To note, the variability in PRP preparation is considered a primary, potential cause of the differential clinical effects of PRP.

Because a commercially available platelet concentration system has not been approved for clinical use in Japan, in this study, autologous PRP was prepared at our institution's Blood Transfusion Service. Classically, for the preparation of platelet concentrates in transfusion, the two major methods for isolating platelets from whole blood are the platelet sedimentation (PS) and BC methods [23]. Principally, both methods consist of a two-stage centrifugation; however, the order of soft and hard spins is different between the two methods. Platelet concentrates isolated from the BC method (hard to soft spins) are less activated during the preparation process [24] and contain fewer WBCs [25], and thus, lower concentrations of proinflammatory cytokines compared to those found using the PS method [23]. Therefore, we utilized the BC method for isolating autologous PRP for intradiscal injection therapy. According to the PRP classification system reported by Dohan, our PRP preparation process is classified as "pure PRP" (P-PRP), which contains fewer WBCs $[8,26]$.

The reparative effect of PRP itself with gelatin hydrogel has been demonstrated in pre-clinical animal studies $[11,12]$. A prospective, randomized, and controlled clinical study to evaluate the effectiveness of intradiscal injection of autologous PRP has recently been reported [27]. Chronic low back pain patients who received intradiscal PRP showed significant improvement in pain and function scores. On the other hand, in this study, only the releasate fraction isolated from activated PRP was injected into the targeted degenerated discs.

Activated platelets are known to release stored intercellular mediators and cytokines from three types of granules: alpha granules, dense granules, and lysosomes [28]. Platelet secretion is dependent on the intensity of activation stimuli (energy-dependent) because lysosomal secretion needs the greatest stimulation, while alpha and dense granules require less stimulation [28]. To standardize the activation process among individuals, a certain amount of $\mathrm{CaCl}_{2}$ and autologous serum was added to PRP for platelet activation. Therefore, the characteristic of this treatment is that the bioactive releasate isolated from activated platelets is directly applied to the cells within degenerated discs.

Importantly, the soluble releasate used in this study could be sterilized via a membrane filter while being injected into the target discs, conferring the advantage of reducing the risk of infection associated with the injection procedure. Second, the soluble releasate can be cryopreserved, affording greater latitude on the timing and number of injections of its use. The presence of thrombin, which is either an allogenic or xenogenic blood product, drudges up concerns about immunogenic reactions or disease transmission in clinical use. Furthermore, thrombin has the potential to degrade proteoglycan in the cartilage tissue [29]. Therefore, we used $\mathrm{CaCl}_{2}$ and autologous serum, instead of human or bovine thrombin, to activate PRP for safe clinical use.

The primary purpose of our study was to assess the safety of PRP releasate; therefore, we aimed to objectively evaluate changes in disc degeneration following PRPreleasate injection by radiographic image analyses. Radiographically, no remarkable progression of disc height narrowing was observed following intradiscal injection of PRP releasate. Although a significant reparative effect on disc height was not observed in this study, as we previously reported [14], our findings suggested that PRP releasate did not negatively impact disc height. The MRI T2-mapping technique may quantitatively evaluate changes in the molecular composition and structural organization of the intervertebral disc [30]. In the present study, we examined whether PRP-releasate injection affects the T2 value of both AF and NP tissues separately using a template-based segmentation method [20]. This method has been developed to segment the AF and NP tissues of a degenerated IVD based on the template of a normal cadaveric lumbar spine. Injected PRP releasate had no effect on T2 values of both AF and NP tissues; these results were similar to those of our previous study on animals [14]. Thus, intradiscal injection of PRP releasate did not negatively affect the matrix of degenerated IVDs.

\section{Conclusions}

In conclusion, we demonstrated that intradiscal injection of PRP releasate to treat patients with low back pain and degenerated IVDs is safe and feasible. This treatment would be especially suitable for young adults and/or premiddle age patients, who generally are not recommended for spinal fusion surgery. Future prospective, doubleblinded, randomized, and placebo-controlled studies will be necessary to determine the efficacy of this treatment. 


\section{Conflict of Interest}

No potential conflict of interest relevant to this article was reported.

\section{Acknowledgments}

The authors would like to thank Dr. Takeshi Matsumoto, Ms. Arisa Sakurai, and other staff members of the Blood Transfusion Service of Mie University Hospital, for their cooperation and assistance in the preparation of PRP. The authors also would like to thank Ms. Mary Ellen Lenz for her assistance in the preparation of the manuscript. This study was supported by grants from the Ministry of Education, Culture, Sports, Science and Technology (Japan).

\section{ORICD}

Koji Akeda: 0000-0001-9468-9387

Kohshi Ohishi: 0000-0001-8136-9907

Koichi Masuda: 0000-0002-5361-4415

Won C. Bae: 0000-0003-2616-0339

Norihiko Takegami: 0000-0002-4677-3450

Junichi Yamada: 0000-0002-3477-0420

Tomoki Nakamura: 0000-0002-1099-7436

Toshihiko Sakakibara: 0000-0003-0942-2237

Yuichi Kasai: 0000-0003-0578-8602

Akihiro Sudo: 0000-0003-0474-4368

\section{References}

1. Cheung KM, Karppinen J, Chan D, et al. Prevalence and pattern of lumbar magnetic resonance imaging changes in a population study of one thousand fortythree individuals. Spine (Phila Pa 1976) 2009;34:93440.

2. Videman T, Nurminen M. The occurrence of anular tears and their relation to lifetime back pain history: a cadaveric study using barium sulfate discography. Spine (Phila Pa 1976) 2004;29:2668-76.

3. Singer AJ, Clark RA. Cutaneous wound healing. N Engl J Med 1999;341:738-46.

4. Peng B, Hao J, Hou S, et al. Possible pathogenesis of painful intervertebral disc degeneration. Spine (Phila Pa 1976) 2006;31:560-6.

5. Garcia-Cosamalon J, del Valle ME, Calavia MG, et al. Intervertebral disc, sensory nerves and neurotroph- ins: who is who in discogenic pain? J Anat 2010;217: 1-15.

6. Alsousou J, Ali A, Willett K, Harrison P. The role of platelet-rich plasma in tissue regeneration. Platelets 2013;24:173-82.

7. Kon E, Filardo G, Di Martino A, Marcacci M. Platelet-rich plasma (PRP) to treat sports injuries: evidence to support its use. Knee Surg Sports Traumatol Arthrosc 2011;19:516-27.

8. Xie X, Zhang C, Tuan RS. Biology of platelet-rich plasma and its clinical application in cartilage repair. Arthritis Res Ther 2014;16:204.

9. Wang SZ, Rui YF, Tan Q, Wang C. Enhancing intervertebral disc repair and regeneration through biology: platelet-rich plasma as an alternative strategy. Arthritis Res Ther 2013;15:220.

10. Chen WH, Liu HY, Lo WC, et al. Intervertebral disc regeneration in an ex vivo culture system using mesenchymal stem cells and platelet-rich plasma. Biomaterials 2009;30:5523-33.

11. Nagae M, Ikeda T, Mikami Y, et al. Intervertebral disc regeneration using platelet-rich plasma and biodegradable gelatin hydrogel microspheres. Tissue Eng 2007;13:147-58.

12. Sawamura K, Ikeda T, Nagae M, et al. Characterization of in vivo effects of platelet-rich plasma and biodegradable gelatin hydrogel microspheres on degenerated intervertebral discs. Tissue Eng Part A 2009; 15:3719-27.

13. Akeda K, An HS, Pichika R, et al. Platelet-rich plasma (PRP) stimulates the extracellular matrix metabolism of porcine nucleus pulposus and anulus fibrosus cells cultured in alginate beads. Spine (Phila Pa 1976) 2006;31:959-66.

14. Obata S, Akeda K, Imanishi T, et al. Effect of autologous platelet-rich plasma-releasate on intervertebral disc degeneration in the rabbit anular puncture model: a preclinical study. Arthritis Res Ther 2012;14:R241.

15. Pfirrmann CW, Metzdorf A, Zanetti M, Hodler J, Boos N. Magnetic resonance classification of lumbar intervertebral disc degeneration. Spine (Phila Pa 1976) 2001;26:1873-8.

16. Ohtori S, Kinoshita T, Yamashita M, et al. Results of surgery for discogenic low back pain: a randomized study using discography versus discoblock for diagnosis. Spine (Phila Pa 1976) 2009;34:1345-8. 
17. Von Korff M, Jensen MP, Karoly P. Assessing global pain severity by self-report in clinical and health services research. Spine (Phila Pa 1976) 2000;25:314051.

18. Roland M, Morris R. A study of the natural history of back pain. Part I: development of a reliable and sensitive measure of disability in low-back pain. Spine (Phila Pa 1976) 1983;8:141-4.

19. Akeda K, Yamada T, Inoue N, Nishimura A, Sudo A. Risk factors for lumbar intervertebral disc height narrowing: a population-based longitudinal study in the elderly. BMC Musculoskelet Disord 2015;16:344.

20. Bae WC, Bydder G, Masuda K. T2 values of human lumbar discs: template-based segmentation and variations with age, sex and level. In: Annual Meeting of Orthopaedic Research Society; 2013 Jan 26-29; San Antonio, TX; Paper 0231.

21. Otsu N. A threshold selection method from graylevel histograms. IEEE Trans Syst Man Cybern 1979; 9:62-6

22. Faul F, Erdfelder E, Lang AG, Buchner A. G*Power 3: a flexible statistical power analysis program for the social, behavioral, and biomedical sciences. Behav Res Methods 2007;39:175-91.

23. Perrotta P, Snyder E. Platelet storage and tranfusion. In: Michelson AD, editor. Platelets. New York: Academic Press; 2002. p.887-905.
24. Fijnheer R, Pietersz RN, de Korte D, et al. Platelet activation during preparation of platelet concentrates: a comparison of the platelet-rich plasma and the buffy coat methods. Transfusion 1990;30:634-8.

25. Chaudhary R, Aggarwal A, Khetan D, Dayal R. Cytokine generation in stored platelet concentrate: comparison of two methods of preparation. Indian J Med Res 2006;124:427-30.

26. Dohan Ehrenfest DM, Rasmusson L, Albrektsson T. Classification of platelet concentrates: from pure platelet-rich plasma (P-PRP) to leucocyte- and platelet-rich fibrin (L-PRF). Trends Biotechnol 2009;27: 158-67.

27. Tuakli-Wosornu YA, Terry A, Boachie-Adjei K, et al. Lumbar Intradiskal Platelet-Rich Plasma (PRP) Injections: A Prospective, Double-Blind, Randomized Controlled Study. PM R 2016;8:1-10.

28. Reed L. Platelet secretion. In: Michelson A, editor. Platelets. New York: Academic Press; 2002. p.181-95.

29. Furmaniak-Kazmierczak E, Cooke TD, Manuel R, et al. Studies of thrombin-induced proteoglycan release in the degradation of human and bovine cartilage. J Clin Invest 1994;94:472-80.

30. Watanabe A, Benneker LM, Boesch C, Watanabe T, Obata T, Anderson SE. Classification of intervertebral disk degeneration with axial T2 mapping. AJR Am J Roentgenol 2007;189:936-42. 